TENDENCIAS DEL COMPORTAMIENTO ESPACIO-TEMPORAL DE DIABETES MELLITUS Y ENFERMEDADES ISQUÉMICAS EN SAN LUIS POTOSÍ, MÉXICO.

\author{
TENDENCIES OF THE SPATIAL AND TEMPORAL BEHAVIOR OF DIABETES MELLITUS AND ISCHEMIC \\ DISEASE IN SAN LUIS POTOSÍ, MÉXICO.
}

Macías Pérez Asaneth Careli ${ }^{*}$, Domínguez Cortinas Gabriela*, Muñiz Carreón Gregoria Patricia*, Cubillas Tejeda Ana Cristina*, Márquez Mireles Leonardo Ernesto*.

* Universidad Autónoma de San Luis Potosí, México.

Citation: Macías Pérez A.C., Domínguez Cortinas G., Muñiz Carreón G.P., Cubillas Tejeda A.C., Márquez Mireles L.E. (2021) Tendencias del comportamiento espaciotemporal de diabetes mellitus y enfermedades isquémicas en San Luis Potosí, México. Revista Salud Pública y Nutrición, 20 (1), 1-11.

Editor: Esteban G. Ramos Peña, Dr. CS., Universidad Autónoma de Nuevo León, Facultad de Salud Pública y Nutrición, Monterrey Nuevo León, México. Copyright: (C2021 Macías Pérez A.C., et al. This is an open-access article distributed under the terms of Creative Commons Attribution License [CC BY 4.0], which permits unrestricted use, distribution, and reproduction in any medium, provided the original author and source are credited.

Competing interests: The authors have declared that no competing interests exist.

DOI: https://doi.org/10.29105/respyn20.1-1

Recibido: 20 de septiembre 2020; Aceptado: 30 de noviembre 2020

Email: gabriela.dominguez@uaslp.mx 


\title{
TENDENCIAS DEL COMPORTAMIENTO ESPACIO-TEMPORAL DE DIABETES MELLITUS Y ENFERMEDADES ISQUÉMICAS EN SAN LUIS POTOSÍ, MÉXICO.
}

\author{
Macías Pérez Asaneth Careli*, Domínguez Cortinas Gabriela*, Muñiz Carreón Gregoria Patricia*, Cubillas \\ Tejeda Ana Cristina*, Márquez Mireles Leonardo Ernesto*. \\ * Universidad Autónoma de San Luis Potosí, México.
}

\section{RESUMEN}

Introducción. Existen factores sociales adversos que aumentan la vulnerabilidad de las poblaciones en la presencia de las enfermedades crónicas no transmisibles, provocando procesos de transición epidemiológica que influyen en el comportamiento epidemiológico de las afecciones. Objetivo: Analizar las tendencias espaciotemporales de diabetes mellitus y enfermedades isquémicas en San Luis Potosí, México, mediante un análisis retrospectivo (1996-2011) de morbilidad, estableciendo sus principales determinantes sociales. Material y Método: Se calcularon tasas de incidencia por 100000 habitantes de diabetes y enfermedades isquémicas durante el periodo 1996-2011, identificando su comportamiento espaciotemporal a nivel estatal y municipal. Fueron evaluadas relaciones multivariadas entre determinantes sociales y morbilidad. Resultados: La diabetes mostró tendencia al aumento en número de casos ( $m=9.49, \mathrm{R} 2=0.2966$ ) en 26 de los 58 municipios del estado, mientras que las enfermedades isquémicas presentaron tendencias positivas en 4 municipios. El comportamiento de ambas enfermedades se asoció significativamente a factores de vulnerabilidad como la condición indígena $(R 2=0.778)$ y la pobreza alimentaria $(R 2=0.753)$. Conclusiones: La región Huasteca concentró la mayor incidencia de diabetes y enfermedades isquémicas y tendencia al aumento. Condiciones como el ser indígena, no derechohabiente de servicios de salud y presentar pobreza alimentaria determinan el comportamiento de estas enfermedades.

Palabras Clave: Diabetes mellitus; enfermedad isquémica; comportamiento espaciotemporal.

\section{ABSTRACT}

Introduction: There are adverse social factors that increase the vulnerability of populations in the presence of chronic noncommunicable diseases, causing processes of epidemiological transition that influence the epidemiological behavior of the diseases. Objective: Analyze patterns and trends of the spatial and temporal behavior of diabetes mellitus and ischemic diseases in San Luis Potosí, Mexico, through a retrospective analysis (1996-2011) of morbidity and its main social determinants. Material and method: Incidence rates per 100000 inhabitants of diabetes and ischemic diseases during the period 1996-2011 were calculated, identifying spatial and temporal behavior patterns and trends at the state and municipal level. Multivariate relationships between social determinants and morbidity rates were assessed. Results: Diabetes showed a significant trend in the increase in new cases $(m=9.49, R 2=0.2966)$ in 26 of the 58 municipalities of the State, while ischemic diseases showed only significant trends to the increase in 4 Municipalities. Trends and spatial and temporal behavior of both diseases was strongly associated with vulnerability factors such as indigenous status ( $R=0.882 ; R 2=0,778)$ and food poverty $(R=0.868, R 2=0.753)$. Conclusions: Huasteca showed greatest morbidity of diabetes and ischemic diseases, with a tendency to increase. Being indigenous and poor significantly determines the behavior of these diseases.

Key words: Diabetes mellitus; ischemic disease; spatial and temporal behavior. 


\section{Introducción}

En los últimos 25 años procesos de transición epidemiológica ocurridos fundamentalmente en países en desarrollo han llevado al aumento de enfermedades crónicas no transmisibles (ENT) que han influido de manera decisiva en el comportamiento epidemiológico de las afecciones que padece la sociedad. Analizando su impacto de manera cronológica, en el 2008, las ENT fueron responsables del $63 \%$ de las muertes en el mundo, para el 2014 el porcentaje aumentó a 70\%, en 2016 a $72 \%$, y en el 2017 alcanzando el $74 \%$ fueron causantes de la defunción de 38 millones de personas (Organización Mundial de la Salud [OMS], 2017). Padecer alguna patología de esta índole no solamente afecta la calidad y esperanza de vida del enfermo, sino que además impacta fuertemente la economía de las familias y de los sistemas sanitarios. Tan solo en América, según el Plan de Acción y Control de Enfermedades, se prevé una derrama económica de más de 46 billones de dólares durante el periodo 2011-2030 para mitigar los daños en la medida posible (Organización Panamericana de la Salud [OPS], 2014).

En México, las ENT explican el 80\% de muertes (Instituto Nacional de Estadística y Geografía [INEGI], 2016), destacando la diabetes mellitus tipo 2 (DMT2) y las enfermedades isquémicas del corazón (EIC) en los primeros lugares de las causas de morbilidad, de muerte prematura y de discapacidad. La Organización Panamericana de la Salud (OPS) recomienda para un adecuado acceso y cobertura en atención sanitaria un producto interno bruto (PIB) de al menos 6\%, no obstante, para la población mexicana este valor apenas alcanza el 2.5\% (Fondo de las Naciones Unidas para la Infancia [UNICEF], 2017). En el estado de San Luis Potosí, los registros de mortalidad por ENT en los últimos diez años muestran incrementos en los decesos por DMT2 de $29.7 \%$ y por EIC de $30.6 \%$, lo que ha colocado a ambas enfermedades dentro de las tres primeras causas de muerte en la Entidad (Sistema Único de Información de Vigilancia Epidemiológica [SUIVE], 2011).

Adicionalmente, los factores sociales adversos aumentan la vulnerabilidad de las poblaciones ante las ENT, luego entonces, las condiciones de pobreza y exclusión social, el bajo nivel de desarrollo humano y la falta de acceso a servicios de salud gratuitos de calidad, entre otros, toman gran relevancia dado que los impactos de las ENT sobre las poblaciones humanas, no sólo dependerán de la naturaleza de la enfermedad per se, sino de la condición de vulnerabilidad social, ambiental, política, cultural y sanitaria, que podría mermar su capacidad de afrontar los riesgos y mitigar los impactos sobre su estado de salud y bienestar (Armas, et al., 2009) (Bener, et al., 2009).

Dicho lo anterior, la presente investigación tiene como objetivo, identificar y analizar patrones y tendencias del comportamiento espaciotemporal de la DMT2 y las EIC mediante un análisis retrospectivo de un periodo de estudio de 16 años (1996-2011), incorporando un conjunto de determinantes sociales relacionados con ambas enfermedades para la cuantificación multivariada de sus pesos de atribución sobre la condición de morbilidad detectada en el estado de San Luis Potosí.

\section{Material y Método}

Descripción del sitio de estudio. El estado de San Luis Potosí se encuentra localizado en la altiplanicie centro de la República Mexicana; cuenta con una superficie de $60546.79 \mathrm{~km}^{2}$ y representa el $3 \%$ del área total del país. Sus límites geográficos son $24^{\circ}$ $29^{\prime} 21^{\circ} 10^{\prime}$ latitud norte y $98^{\circ} 20^{\prime} 102^{\circ} 18^{\prime}$ longitud oeste, colindando al norte con el estado de Coahuila, noreste con Nuevo León y Tamaulipas, al este con Veracruz, al sur con Hidalgo, Querétaro y Guanajuato, al suroeste con Jalisco y al oeste con Zacatecas (Instituto Nacional de Estadística Geografía e Informática [INEGI], 2015). Está dividido en 58 municipios distribuidos en cuatro regiones geográficas: Altiplano (15 municipios$25.86 \%$ ), Centro (11 municipios-18.96\%), Media (12 municipios-20.68\%) y Huasteca (20 municipios$34.48 \%$ ) (Servicios de Salud del estado de San Luis Potosí, 2018). Según el Censo de Población y Vivienda 2015, el Estado cuenta con un total de 2585 518 habitantes, de los cuales $392999(15.2 \%)$ son indígenas, cuya mayor concentración geográfica se da en la región Huasteca (83\%); cabe señalar que la población indígena que habita en el estado de San Luis Potosí representa el $10.7 \%$ del total en el país (Instituto Nacional de Estadística Geografía e Informática [INEGI], 2015). Según fuentes oficiales, el $50.5 \%$ de la población total del Estado se encuentra en condición de pobreza ya sea moderada $(37.7 \%)$ o extrema (12.8\%) (Consejo Nacional de 
Población [CONAPO], Secretaria de Salud [SSA], Secretaría de Gobernación [SEGOB], 2014).

Tratamiento y análisis de los datos. A partir de los Servicios de Salud del Estado, fueron obtenidos los reportes del número de casos nuevos de DMT2 y EIC por semana epidemiológica, considerando un periodo de 16 años comprendidos entre 1996 a 2011 y con un nivel de desagregación municipal. Los datos fueron analizados mediante el software SPSS versión 25.0. Con fines de comparabilidad estadística interanual e intermunicipal para cada enfermedad según año y municipio de estudio, se calcularon las tasas de incidencia acumulada por 100000 habitantes utilizando los datos de población de censos, conteos y proyecciones del estado de San Luis Potosí, proporcionados por el Instituto Nacional de Estadística y Geografía (INEGI). Para la identificación de patrones de comportamiento espacial y temporal de DMT2 y EIC, así como de sus principales tendencias durante el periodo de estudio, todos los datos de incidencia acumulada fueron analizados a través de un modelo de tendencia lineal aplicando el método de mínimos cuadrados. La construcción cuantitativa de las relaciones multivariadas entre los determinantes sociales y las tasas de morbilidad de DMT2 y EIC, se realizó a través de un análisis exploratorio de factores aplicando el método de extracción de Componentes Principales (CP) mediante el programa estadístico PASW Statistics versión 18. Se aplicaron pruebas de viabilidad y pertinencia estadística del CP tales como: la determinante de la matriz, la prueba de esfericidad de Bartlett y la prueba de Káiser-MeyerOlkin (KMO) (Meyers, Gams, Guarino, 2006) (Hair, Anderson, Tatham, Black, 2007). Se generó la tabla de la varianza total explicada para identificar el número de componentes con eigenvalores mayores que 1 y se trabajó con una matriz de componentes rotada por el método varimax para facilitar la interpretación de las cargas que los indicadores tienen en los componentes extraídos. (Meyers, Gams, Guarino, 2006) (Hair, Anderson, Tatham, Black, 2007). Los determinantes sociales seleccionados para este análisis fueron: 1) Habitantes por unidades de consulta externa (HAUCE), 2) Unidades de hospitalización (UNHOSP), 3) Habitantes por personal médico (HAPEME), 4) \% Población sin derechohabiencia a servicios de salud (PSINDH), 5) \% Población con pobreza alimentaria (PPOBRA), 6) \% Población con pobreza de capacidades (PPOBRC), 7) \% Población con pobreza patrimonial (PPOBRP), 8) \% Población analfabeta de 15 años y más (PPOBAN), 9) \% Población con hacinamiento (PHACIN), 10) \% Población indígena (PPOBIN), 11) \% Población ocupada con hasta 2 salarios mínimos (PPO2SM), 12) Región Estatal (REGN). Todos los datos de los determinantes sociales seleccionados para los años 2000, 2005 y 2010 con desagregación municipal fueron obtenidos de INEGI, Consejo Nacional de Población (CONAPO) y Consejo Nacional de Evaluación de la Política de Desarrollo Social (CONEVAL).

\section{Resultados}

Tasas de incidencia acumulada y análisis de tendencia en el Estado.

La tasa de incidencia acumulada de DMT2 de 19962011 sumó 6036.8 nuevos casos, con una media anual de 377.3; ocho años superaron la media (2000, 2004-2010). La tasa más alta fue vista en el año 2000 con 527.6 y la más baja en 1996 con 211.4. El comportamiento de la incidencia tuvo variaciones, observándose aumentos sostenidos de 1996-1998 y 2004-2006; y un decremento del 2007-2009. La DMT2 durante el periodo evaluado mostró una tendencia al aumento de la enfermedad estadísticamente significativa $(\mathrm{m}=9.49, \mathrm{R} 2=0.2966)$, a una razón estimada de 9 casos nuevos por 100000 habitantes/año y de 152 casos nuevos en 16 años (Figura 1A). En cuanto a EIC, la tasa de incidencia acumulada fue de 806.0 con una media de 50.4; ocho años la superaron: 2001, 2003 y 2005-2010. La tasa más elevada ocurrió en 2007 con 93.0 y la más baja en 1996 con 38.1. Aumentos sostenidos de tres años tuvieron lugar de 1999-2001 y del 2005-2007. Un decremento constante fue observado del 2008-2011. La tendencia mostró un aumento no significativo de la patología $(\mathrm{m}=0.7873, \mathrm{R} 2=0.1986)$ con un nuevo caso por 100000 habitantes/año (Figura 1B). De manera general, la Huasteca fue la zona más afectada, representando el $40.17 \%$ de la tasa de incidencia acumulada de diabetes y el $34.68 \%$ de las cardiopatías isquémicas.

Tasa de incidencia acumulada por municipio. DMT2. Ciudad Valles (9 998.6), Cárdenas (7 191.9), San Luis Potosí (7 007.8) y El Naranjo (6 748.8) registraron las mayores tasas de incidencia, mientras que Cerro de San Pedro (737.4), Villa de Ramos (1 
086.2) y Villa de Arista (1 249.5) presentaron las más bajas. La media fue de 3447.3 nuevos casos por 100 000 habitantes, superando esta cifra 23 de los 58 municipios (40.0\%) del Estado, de los cuales el 57\% pertenecen a la Huasteca, $26 \%$ a la región Media, $13 \%$ a la Centro y $4 \%$ al Altiplano. Al comparar las tasas de incidencia de DMT2 del municipio más afectado con el menos afectado, la diferencia resulta 7.5 veces mayor. EIC. Ciudad Valles (1 573.4), Cerritos (1 459.6) y San Luis Potosí (1 259.0) registraron las tasas de incidencia más altas, mientras que cerro de San Pedro (0.0), Mexquitic (8.5) y Ciudad Fernández (9.8) mostraron las más bajas. La media fue de 256.6 siendo superada por 15 de 58 municipios (26\%), de los cuales el $47 \%$ corresponden a la Huasteca, 26\% al Altiplano, 20\% a la región Media y 7\% a la región Centro (Tabla 1).

Análisis de Tendencia Municipal.

DMT2. Partiendo de la estimación lineal de 19962011 a nivel municipal, se detectaron tendencias significativas al aumento en 26 municipios del Estado (45\%) de los cuales el 38\% (10/26) se concentra en la región Huasteca, 23\% (6/26) en la región Altiplano, 23\% (6/26) en la Media y 15\% (4/26) en la Centro. Los municipios con las mayores tasas de incremento de la enfermedad fueron Tamuín $(\mathrm{m}=33.8, \quad \mathrm{R} 2=0.4704), \quad \mathrm{El}$ Naranjo $\quad(\mathrm{m}=29.8$, $\mathrm{R} 2=0.3950) \quad \mathrm{y}$ Lagunillas $(\mathrm{m}=29.1, \quad \mathrm{R} 2=0.7272)$ (Tabla 1, Figura 2A).

EIC. A partir de la estimación lineal, 9 municipios (16\%) se identificaron con tendencias significativas: 5 a la disminución y 4 al incremento; de los 4 con tendencia al aumento de nuevos casos, 3 se localizan en la Huasteca: Tamuín $(m=3.9, R 2=0.6935)$, San Martín Chalchicuautla $(\mathrm{m}=0.87, \mathrm{R} 2=0.571)$ y Ciudad Valles $(\mathrm{m}=8.5, \mathrm{R} 2=0.3145)$ y uno en región Media: Rioverde ( $m=3.2, \mathrm{R} 2=0.6907)$ (Tabla 1, Figura 2B).

Análisis multivariado de la morbilidad y los determinantes sociales de la salud.

Los resultados de viabilidad y pertinencia estadística del análisis de CP fueron los siguientes: a) La determinante de la matriz de correlación mostró un alto grado de colinealidad entre las variables analizadas (determinante $=2.72 \mathrm{E}-010$ ) siendo significativamente diferente de la matriz identidad; b) La prueba de esfericidad de Bartlett fue significativa $\quad(\mathrm{Chi} 2=1141.62 ; \quad \mathrm{gl}=78 ; \quad \mathrm{p}<0.0001)$ indicando alta correlación entre variables; y c) La prueba KMO de adecuación de muestreo fue de 0.789 , sugiriendo que la naturaleza de los datos es adecuada para el tipo de análisis factorial sugerido (Meyers, Gams, Guarino, 2006).

Los resultados del análisis de $\mathrm{CP}$ mostraron un porcentaje de varianza total explicada de $80.9 \%$ extraída en los primeros tres componentes, de los cuales el primero explica el $53.0 \%$ de la varianza, el segundo $18.7 \%$ y el tercero $9.25 \%$ (Tabla 1 ). La matriz de componentes rotada mostró las variables que presentaron los mayores coeficientes de correlación (R) en cada uno de los componentes extraídos reflejando la intensidad y peso de atribución de cada uno de ellos (Tabla 1). A partir de dichos coeficientes se obtuvieron los valores de R2 que representan la fuerza de la asociación de cada determinante social (variables independientes) con la condición de morbilidad de DMT2 y EIC (variable dependiente-componentes 1 y 2 ) identificada en el Estado (Figura 3).

La integración de los resultados del análisis multivariado permitió determinar que en el estado de San Luis Potosí el comportamiento espaciotemporal de ambas enfermedades se encuentra significativamente asociado a factores de vulnerabilidad como: pertenecer a una población indígena $(\mathrm{R}=0.882 ; \mathrm{R} 2=0.778)$, padecer pobreza alimentaria $(\mathrm{R}=0.868, \quad \mathrm{R} 2=0.753)$, pobreza de capacidades $(\mathrm{R}=0.858, \quad \mathrm{R} 2=0.736)$ y pobreza patrimonial $(\mathrm{R}=0.815, \mathrm{R} 2=0.664)$, habitar en la región Huasteca $(\mathrm{R}=0.797, \mathrm{R} 2=0.635)$, presentar condición de hacinamiento $(\mathrm{R}=0.695, \mathrm{R} 2=0.483) \mathrm{y}$ carecer de acceso a los sistemas de salud gratuita $(\mathrm{R}=0.665, \mathrm{R} 2=0.442)$ (Tabla 2 y Figura 3 ). Dichos factores sociales en su conjunto estarían explicando poco más del $50 \%$ del comportamiento espaciotemporal de la carga de morbilidad por DMT2 y EIC en esta Entidad. Aunque con menor peso explicativo (19\%) el conjunto de factores referentes a la prestación de servicios del sistema de salud del Estado tales como el número de unidades de consulta externa y de hospitalización, también estarían jugando un papel importante en el comportamiento espacial y temporal de ambas enfermedades (HAUCE, $\mathrm{R}=-0.912, \quad \mathrm{R} 2=0.831$; UNHOSP, R=-0.659, R2=0.434) (Tabla 2 y Figura $3)$. 
Tabla 1. Tasas de incidencia acumulada y tendencias de DMT2 y EIC a nivel de desagregación municipal durante periodo de 1996 a 2011

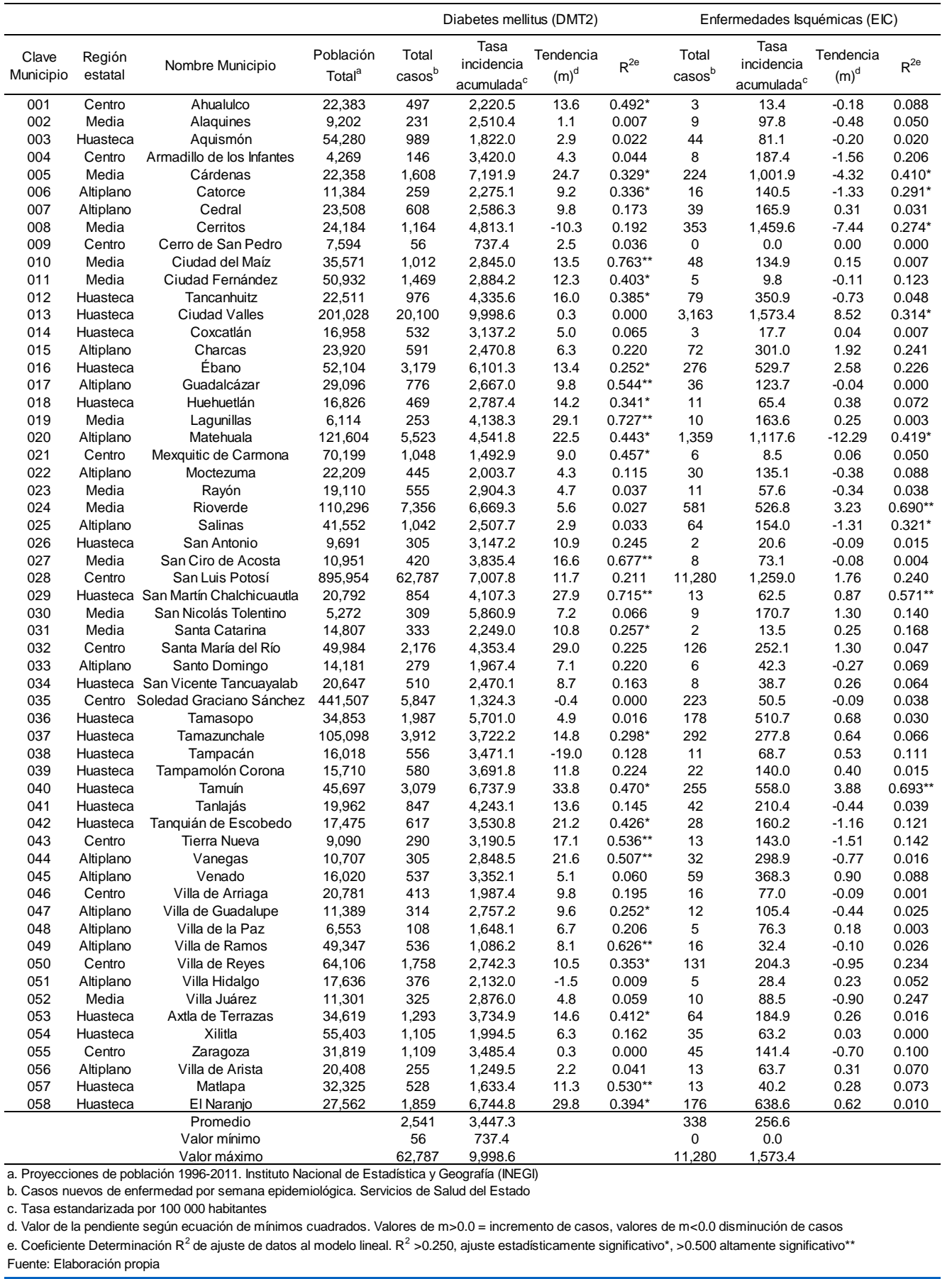



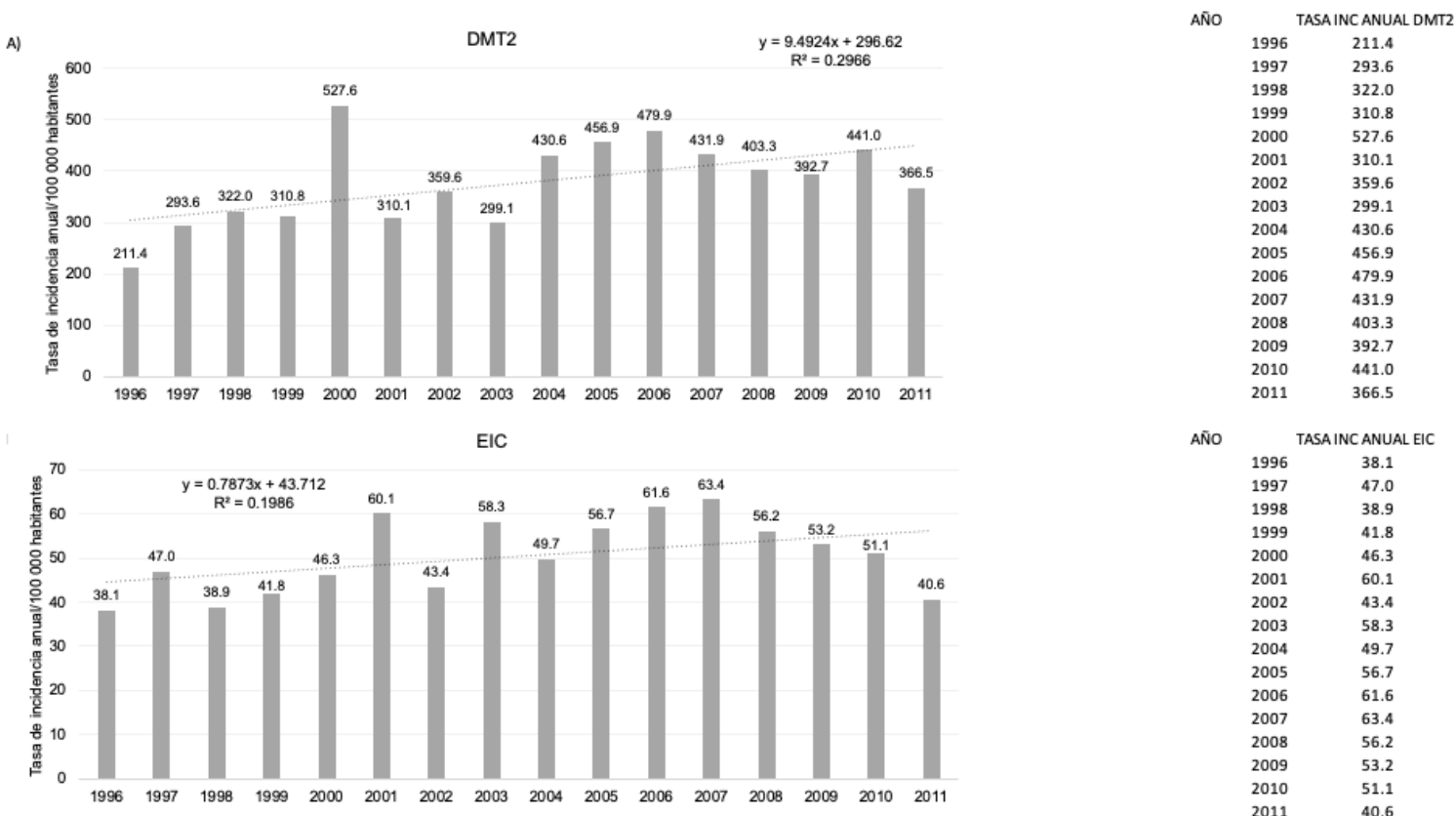

AÑO

$\begin{array}{cc} & \text { TASAINC ANUAL EIC } \\ 1996 & 38.1 \\ 1997 & 47.0 \\ 1998 & 38.9 \\ 1999 & 41.8 \\ 2000 & 46.3 \\ 2001 & 60.1 \\ 2002 & 43.4 \\ 2003 & 58.3 \\ 2004 & 49.7 \\ 2005 & 56.7 \\ 2006 & 61.6 \\ 2007 & 63.4 \\ 2008 & 56.2 \\ 2009 & 53.2 \\ 2010 & 51.1 \\ 2011 & 40.6\end{array}$

Fuente: Elaboración propia

Figura 1. Comportamiento de las tasas de incidencia anual/100 000 hab de DMT2 (A) y EIC (B) en el periodo 1996 a 2011 en el estado de San Luis Potosí, México.

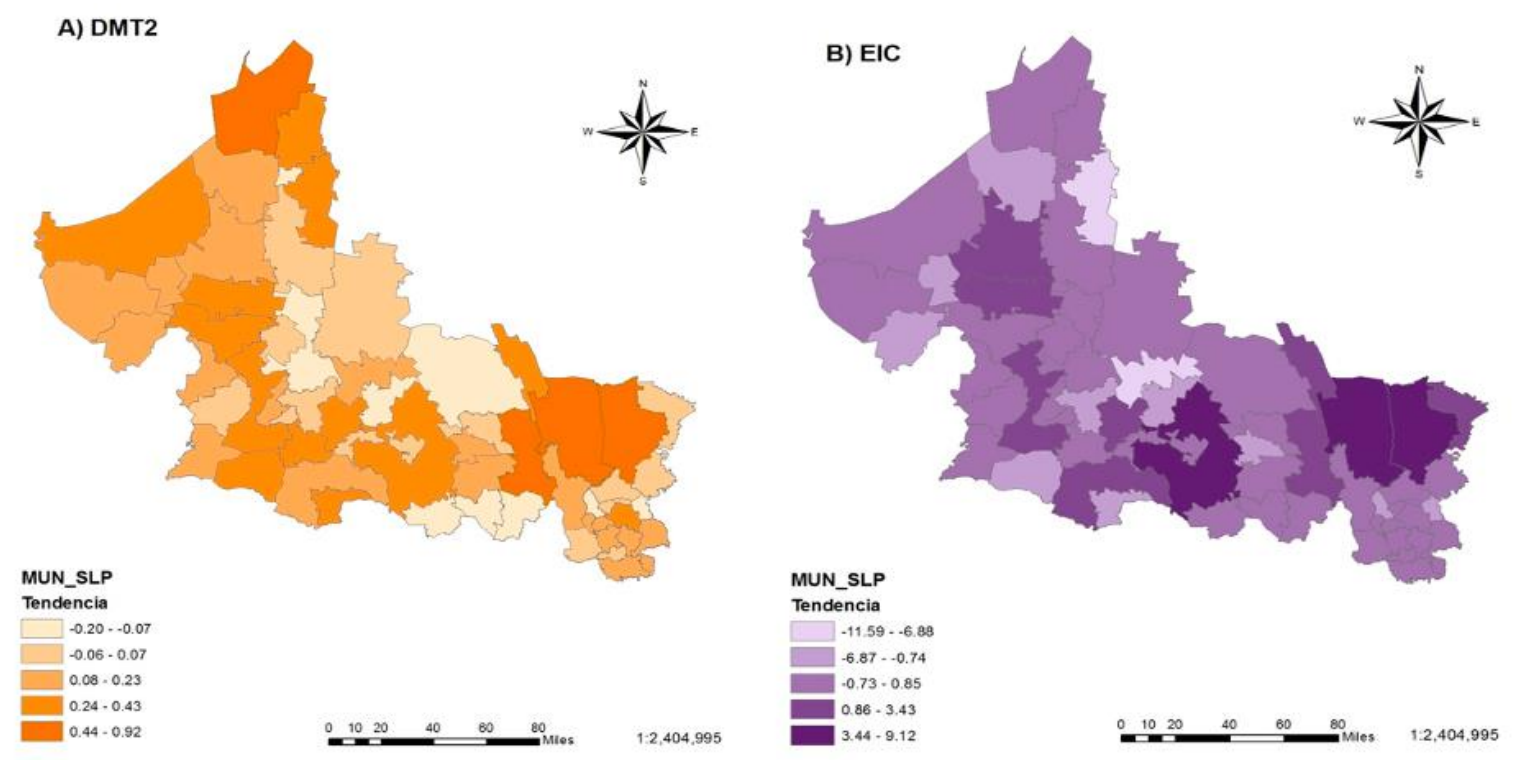

Fuente: Elaboración propia

Figura 2. Comportamiento espacial de las tendencias de DMT2 y EIC a nivel de desagregación municipal de 1996 a 2011 en el estado de San Luis Potosí 
Diabetes mellitus; enfermedad isquémica;

Tabla 2. Autovalores y varianza acumulada por componente (A), y matriz rotada con saturación (correlación) por componente extraído y variable observada (determinantes sociales) (B).

A) Componente

\begin{tabular}{cccc} 
& \multicolumn{3}{c}{ Autovalores iniciales } \\
\hline & Total & \% varianza explicada & \% varianza acumulada \\
1 & 7.422 & 53.016 & 53.02 \\
2 & 2.619 & 18.708 & 71.72 \\
3 & 1.296 & 9.258 & 80.98 \\
\hline
\end{tabular}

B) Matriz de Componentes rotada

Variables observadas (determinantes sociales)

$\%$ Población indígena

$\%$ Población con pobreza alimentaria

$\%$ Población con pobreza de capacidades

\% Población con pobreza patrimonial

Región estatal

$\%$ Población con hacinamiento

\% Población sin derechohabiencia

Habitantes por unidades de consulta externa

\% población ocupada con hasta 2 salarios mínimos

Unidades de hospitalización

\% Población analfabeta

Habitantes por personal médico

Método de extracción: Análisis de componentes principales

Método de rotación: Normalización Varimax con Kaiser
Componentes extraídos

$\begin{array}{lll}1 & 2 & 3\end{array}$

$\begin{array}{lll}0.882 & 0.135\end{array}$

$\begin{array}{lll}0.868 & 0.411 & -0.186\end{array}$

$\begin{array}{lll}0.858 & 0.415 & -0.206\end{array}$

$\begin{array}{lll}0.815 & 0.418 & -0.234\end{array}$

$\begin{array}{lll}0.797 & -0.166 & 0.305\end{array}$

$\begin{array}{lll}0.695 & 0.345 & -0.255\end{array}$

$\begin{array}{lll}0.665 & 0.395 & -0.301\end{array}$

$-0.160-0.912$

$\begin{array}{lll}0.504 & 0.789 & -0.185\end{array}$

$\begin{array}{lll}-0.145 & -0.659 & 0.549\end{array}$

$0.558 \quad 0.579$ $-0.289-0.852$

\section{Fuente: Elaboración propia}

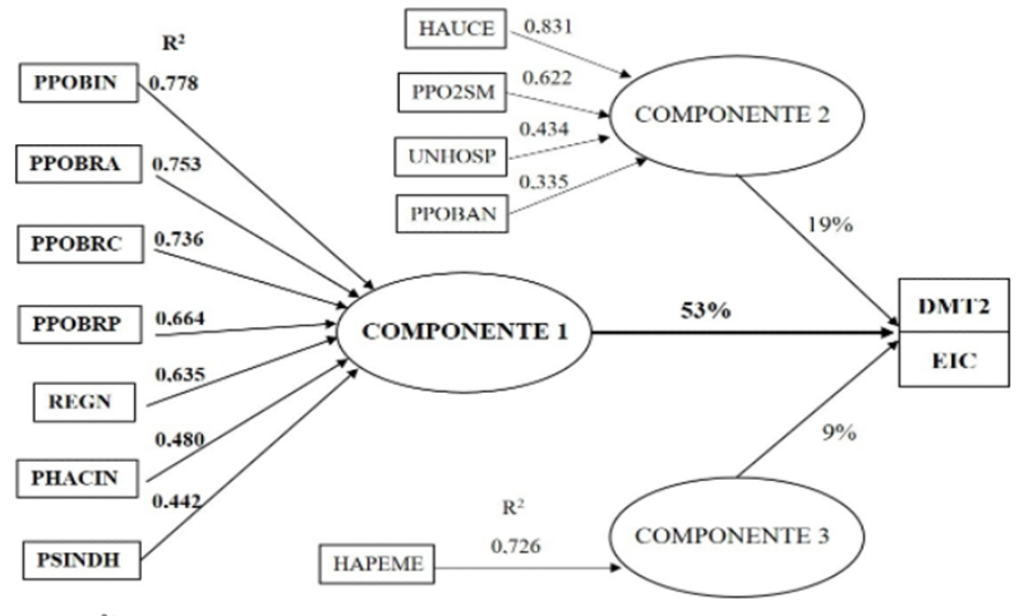

Valores $\mathrm{R}^{2 \text { - }}$ nivel de asociación multivariada entre fracción explicada por variable y componente de mayor saturación

Habitantes por unidades consulta externa (HAUCE), Unidades hospitalización (UNHOSP), Habitantes por personal médico (HAPEME), Pob sin derechohabiencia (PSINDH), Pob pobreza alimentaria (PPOBRA), Pob pobreza capacidades (PPOBRC), Pob pobreza patrimonial (PPOBRP), Pob analfabeta (PPOBAN), Pob hacinamiento (PHACIN), Pob indigena (PPOBIN), Pob ocupada con 2 salarios mínimos (PPO2SM), Región Estatal (REGN)

Fuente: Elaboración propia

Figura 3. Modelo de asociaciones ponderadas entre las variables observadas (determinantes) y componentes extraídos 


\section{Discusión}

En San Luis Potosí las enfermedades crónicas han estado presentes en los primeros lugares de morbilidad desde 1985 (Secretaría de Salud [SSA], 2017). En la actualidad el problema se acentúa ya que las EIC y DMT2 representan la primera y segunda causa de mortalidad en el Estado siendo responsables del 43\% de las defunciones (Instituto Nacional de Estadística y Geografía [INEGI], 2017) (Instituto Nacional de Estadística y Geografía [INEGI], 2018). La menor incidencia de DMT2 y EIC fue registrada en 1996 y aunque en los 15 años subsecuentes se observaron fluctuaciones, las tasas de incidencia en la mayoría de los años fueron significativamente mayores a las de 1996. Este comportamiento pudiera estar ligado a la política de descentralización de los servicios de salud durante el sexenio de Ernesto Zedillo (1994-2000) iniciando en 1995 y consolidando a cada Estado como organismo público descentralizado en 1997 (López, 2012) (Organización Panamericana de la Salud [OPS], 2018). La adaptación a esta nueva condición de los servicios de salud, así como la afiliación masiva de población al Seguro Popular entre 2000 y 2006 pudieron contribuir a una mayor capacidad de registro de casos de enfermedad en los años analizados (Flamand, Moreno, 2015) (Instituto Nacional de Salud Pública [INSP], 2013) (Secretaría de Salud [SSA], 2019). No obstante, las tendencias emanadas del análisis histórico de ambas enfermedades muestran un comportamiento ascendente en el Estado con énfasis en las regiones Media y Huasteca, que además de presentar la mayor carga de enfermedad en el periodo estudiado, también concentran el $87 \%$ de la población indígena y más del $52 \%$ de población que vive en condiciones de pobreza, hacinamiento, bajos ingresos, bajo nivel educativo y sin derechohabiencia (Instituto Nacional de Estadística Geografía e Informática [INEGI], 2015) (Consejo Nacional de Población [CONAPO], Secretaria de Salud [SSA], Secretaría de Gobernación [SEGOB], 2014).

Este panorama es preocupante si consideramos que no sólo la DMT2 y las EIC van en aumento, sino que algunos de sus principales determinantes sociales tendrán muy poca o nula mejoría en el Estado según ha estimado CONAPO en sus proyecciones de marginación y pobreza a 2030 (Consejo Nacional de Población [CONAPO], Secretaria de Salud [SSA], Secretaría de Gobernación [SEGOB], 2014). Las tendencias estimadas en nuestro estudio mostraron coherencia con datos publicados en la ENSANUT (Instituto Nacional de Salud Pública [INSP], 2013) que ha reportado para el Estado un incremento de DMT2 y EIC del $3.8 \%$ y $2.2 \%$ respectivamente en el periodo 2006-2012. Asociaciones multivariadas detectadas en nuestro modelo de CP han sido también reportadas por otros autores que refieren mayores prevalencias de DMT2 y EIC en poblaciones con bajo nivel educativo, bajo nivel de ingresos y sin acceso a servicios de salud gratuitos (Armas, et al., 2009) (Bener, et al., 2009).

Es importante expresar que, si bien, los determinantes evaluados mostraron un alto peso explicativo de las incidencias de ambas ENT en la entidad, estos no son los únicos que pudieran estar regulando dichos comportamientos, tal como reportan otros autores que han identificado determinantes de suma importancia para DMT2 y EIC como edad, sexo, sedentarismo, sobrepeso, obesidad y dieta (Escolar, 2009) (Hu, et al., 2003) (Périssé, De Andrade, Caminha, 2010) (Ramos, et al., 2012) (Bacallao, Díaz-Perera, Alemañy, 2012). La carencia de información oficial de dichos determinantes, así como la agregación de la información disponible a nivel de población general, impidió la incorporación de estos indicadores al análisis estadístico, lo que representa una limitante en nuestro estudio. Sin embargo, los determinantes sociales con mayor atribución en el comportamiento de estas ENT referidas en el primer componente del análisis multivariado da pauta para dirigir intervenciones con enfoque territorial, social y epidemiológico.

\section{Conclusiones}

El análisis espacial y temporal del comportamiento mostrado por las ENT a nivel no solo estatal, sino también, regional y local, permiten la identificación de áreas prioritarias a intervenir. Es necesario entonces, no sólo diagnosticar tempranamente y atender los daños causados por ambas enfermedades, sino que, a través de la contextualización y concientización de la sociedad respecto a la problemática, se promuevan cambios en los estilos de vida actuales que tanto contribuyen con este grave y creciente problema de salud pública. 


\section{Agradecimientos}

El presente estudio fue financiado por el Fondo Sectorial de Investigación Ambiental de la Secretaría del Medio Ambiente y Recursos Naturales (SEMARNAT)_Consejo Nacional de Ciencia y Tecnología (CONACYT) 2014-2017 en México, a quien agradecemos el apoyo brindado, que hizo posible su realización.

\section{Bibliografía}

Organización Mundial de la Salud (OMS). (2017). 10 datos sobre la situación sanitaria mundial. Recuperdo de: http://www.who.int/features/factfiles/global bur den/es/

Organización Panamericana de la Salud (OPS). (2014). Plan de acción para la prevención y el control de las enfermedades no transmisibles en las Américas 2013-2019. Washington, DC: OPS. Recuperado de: https://www.paho.org/hq/index.php?option=com _content\&view=article\&id=14960:ultimosindicadores-basicos-de-la-ops-arrojan-luz-sobrela-situacion-de-salud-en-lasamericas \&Itemid $=1926 \&$ lang $=e s$

Instituto Nacional de Estadística y Geografía (INEGI). (2016). Principales causas de mortalidad por residencia habitual, grupos de edad y sexo del fallecido. Recuperado de: https://www.google.com/search?client=safari\&rl $\mathrm{s}=\mathrm{en} \& \mathrm{q}=1 .+\mathrm{http}: / / \mathrm{www}$.inegi.org.mx/est/conteni dos/proyectos/registros/vitales/mortalidad/tabula dos/ConsultaMortalidad asp\&ie $=$ UTF-

8\&oe=UTF-8Institute for Health Metrics and Evaluation (IHME). GBD Compare, México [Acceso el 09 de septiembre del 2017]. Disponible en: http://www.healthdata.org/mexico

Fondo de las Naciones Unidas para la Infancia (UNICEF). (2019). Inversión en la Infancia en América Latina y el Caribe: Una estrategia para acelerar el logro de los Objetivos de Desarrollo Sostenible. Recuperado de: https://www.unicef.org/lac/sites/unicef.org.lac/fi les/2019-03/PPT-

Panel\%202.2_02_Hector\%20Villarreal.pdf
Sistema Único de Información de Vigilancia Epidemiológica (SUIVE), Dirección General de Epidemiología (DGE), Secretaria de Salud (SSA). (2011). Información Epidemiológica de Morbilidad, Versión ejecutiva, Anuario 2011, México, Distrito Federal.

Instituto Nacional de Estadística Geografía e Informática (INEGI). (2015). Anuario estadístico y geográfico de San Luis Potosí, México. Recuperado de: http://www.inegi.gob.mx

Servicios de Salud del estado de San Luis Potosí. (2019). Diagnóstico Sectorial Sector Salud San Luis Potosí 2018. Recuperado de: http://www.slpsalud.gob.mx/transparenciaadmo n/transparencia/2019/ene/DiagnosticoSalud/DIA GNOSTICO-SALUD.pdf

Consejo Nacional de Población (CONAPO), Secretaria de Salud (SSA), Secretaría de Gobernación (SEGOB). (2014). Dinámica demográfica 1990-2010 y proyecciones de población 2010-2030. San Luis Potosí Primera edición.

Meyers LS, Gamst G, Guarino AJ. (2006). Applied Multivariante Research. Design and Interpretation. E.U.A.: SAGE Publications.

Hair J, Anderson R, Tatham R, Black W. (2007). Análisis multivariante. 5a ed. Madrid: PrenticeHall.

Secretaría de Salud (SSA). (2017). Anuarios de Morbilidad 1984 - 2017. Dirección General de Epidemiología - Dirección General Adjunta de Epidemiología Recuperado de: https://www.gob.mx/salud/acciones-yprogramas/anuarios-de-morbilidad-1984-2017

Instituto Nacional de Estadística y Geografía (INEGI). (2018). Características de las Defunciones Registradas en México durante $2017 . \quad$ Recuperado de: https://www.inegi.org.mx/contenidos/saladepren sa/boletines/2018/EstSociodemo/DEFUNCION ES2017.pdf

Instituto Nacional de Estadística y Geografía (INEGI). (2017). Estadísticas a propósito del día 
de muertos (2 de noviembre) Datos nacionales. Recuperado de: http://www.inegi.org.mx/saladeprensa/aproposit o/2017/muertos2017_Nal.pdf

López-García EG. (2012). La descentralización de los servicios de salud. Biblioteca Jurídica Virtual del Instituto de Investigaciones Jurídicas de la UNAM. Recuperado de: http://historico.juridicas.unam.mx/publica/librev /rev/arsiu/cont/47/act/act6.pdf

Organización Panamericana de la Salud (OPS). (2018). Antología de la Atención a la Salud en México. Recuperado de: https://www.paho.org/mex/index.php?option=co m_content\&view=article \&id=204:antologiaatencion-salud-mexico\&Itemid $=315$

Flamand L, Moreno-Jaimes C. (2015). La protección social en salud durante el gobierno de Calderón. Avances y rezagos en el diseño y la implementación del Seguro Popular (20062012). Foro internacional, 55(1), 217-261. Recuperado de: http://www.scielo.org.mx/scielo.php?script=sci_ arttext\&pid=S0185-

013X2015000100217\&lng=es\&tlng=es.

Instituto Nacional de Salud Pública (INSP) [página electrónica]. (2013). México: Encuesta Nacional de Salud y Nutrición 2012. Resultados por entidad federativa. Recuperado de: http://ensanut.insp.mx/informes/SanLuisPotosiOCT.pdf

Secretaría de Salud (SSA), Comisión Nacional de Protección Social en Salud, Comunicación y Protección Social. (2019). Información general del Sistema Nacional de Protección Social en Salud. Recuperado de: http://www.salud.gob.mx/transparencia/inform adicional/InfoGralSP.pdf

Armas Rojas, Nurys B., Dueñas Herrera, Alfredo, de la Noval García, Reinaldo, Castillo Guzmán, Antonio, Suárez Medina, Ramón, Varona Perez, Patricia, \& Bonet Gorvea, Mariano. (2009). Enfermedades del corazón y sus características epidemiológicas en la población cubana de 15 años y más. Revista Cubana de Investigaciones
Biomédicas, 28(4). Recuperado en 22 de abril de 2020, de http://scielo.sld.cu/scielo.php?script=sci_arttext \&pid=S0864$\underline{03002009000400004 \& \operatorname{lng}=\mathrm{es} \& \ln \mathrm{ln}=\mathrm{es} .}$.

Bener A, Zirie M, Ibrahim M, Janahi IM, Al-Hamaq A, Musallam M, Wareham NJ, et al. (2009). Prevalence of diagnosed and undiagnosed diabetes mellitus and its risk factors in a population-based study of Qatar. Diabetes research and clinical practice. 84:99-106. doi.org/10.1016/j.diabres.2009.02.003

Escolar-Pujolar A. (2009). Determinantes sociales frente a estilos de vida en la diabetes mellitus de tipo 2 en Andalucia: la dificultad para llegar a fin de mes o la obesidad?. Gaceta Sanitaria, 23(5), 427-432. Recuperado en 23 de abril de 2020 , de http://scielo.isciii.es/scielo.php?script=sci_arttex t\&pid=S0213$\underline{91112009000500012 \& \operatorname{lng}=\mathrm{es} \& \ln g=\mathrm{es}}$.

Hu FB, Li TY, Colditz GA, Willett WC, Manson JE, et al. (2003). Television watching and other sedentary behaviors in relation to risk of obesity and type 2 diabetes mellitus in women. JAMA. 289(14):1785-1791.

Périssé, Germana, Medronho, Roberto de Andrade, \& Escosteguy, Claudia Caminha. (2010). Espacio urbano y la mortalidad por enfermedad isquémica del corazón en adultos mayores en Rio de Janeiro. Arquivos Brasileiros de Cardiologia, 94(4), 463-471. Epub March 05, 2010.https://doi.org/10.1590/S0066782X2010005000009

Ramos LE, Gallardo U, Cabrera J, Salgado L, Adam D, Mahía M. (2012). Atherogenic risk and heart diseases in middle-aged women. Instituto Nacional de Angiología y Cirugía Vascular. Revista Cubana de Angiología y Cirugía Vascular;13(2).

Alvarez-Cortés JT, Bello-Hernández V, PérezHechavarría G, Antomarchi-Duany O, BolívarCarrión ME. (2013). Factores de riesgo coronarios asociados al infarto agudo del miocardio en el adulto mayor. MEDISAN, 17(1), 
54-60. Recuperado en 22 de abril de 2020, de http://scielo.sld.cu/scielo.php?script=sci_arttext \&pid=S1029-

$30192013000100008 \& \ln g=e s \& t \operatorname{lng}=$ es.

Bacallao Gallestey, Jorge, Díaz-Perera Fernández, Georgia, \& Alemañy Pérez, Eduardo. (2012). Patrones de concentración social de factores de riesgo aterosclerótico y enfermedades del corazón en La Habana. Revista Cubana de Salud Pública, 38(4), 511-524. Recuperado en 22 de abril de 2020, de http://scielo.sld.cu/scielo.php?script=sci arttext $\underline{\text { \&pid=S086434662012000400003\&lng=es\&tlng }}$ =es. 